\title{
Injectivity of the spherical means operator
}

\author{
Alexander G. Ramm ${ }^{\text {a,b }}$ \\ a LMA-CNRS, 31 Chemin J.Aiguier, Marseille 13402, France \\ b Mathematics Department, Kansas State University, \\ Manhattan, KS 66506-2602, USA \\ E-mail address: ramm@math.ksu.edu
}

(Reçu le 4 October 2002, accepté le 4 October 2002)

Abstract. Let $S$ be a surface in $\mathbb{R}^{n}$ which divides the space into two connected components $D_{1}$ and $D_{2}$. Let $f \in C_{0}\left(\mathbb{R}^{n}\right)$ be some real-valued compactly supported function with supp $f \subset$ $D_{1}$. Consider

$$
M f:=m(y, r):=\int_{\mathbb{R}^{n}} f(z) \delta(|y-z|-r) d z,
$$

where $\delta$ is the delta-function, $y \in S$ and $r>0$ are arbitrary. A general, local at infinity, condition on $S$ is given, under which $M$ is injective, that is, $M f=0$ implies $f=0$. The injectivity result is extended to the case when the Fourier transform of $f$ is quasianalytic, so that compactness of support of $f$ is not assumed. A sufficient condition on $S$ is given, under which $M^{-1}$ can be analytically constructed. Two examples of inversion formulas are given: when $S$ is a plane, and when $S$ is a sphere. These formulas can be used in applications. (C) 2002 Académie des sciences/Éditions scientifiques et médicales Elsevier SAS

\section{Injectivité de l'operateur de moyen spherique}

Résumé. $\quad$ Soit $S$ une surface de $\mathbb{R}^{n}$ qui divise l'espace en deux composantes connectées $D_{1}$ and $D_{2}$. Soit $f \in C_{0}^{\infty}\left(\mathbb{R}^{n}\right)$ une fonction à valeurs réeles, supp $f \subset D_{1}$. Considérons

$$
M f:=m(y, r):=\int_{\mathbb{R}^{n}} f(z) \delta(|y-z|-r) d z,
$$

où $\delta$ est la delta-fonction, $y \in S$ et $r>0$ sont quelconques. Une condition générale, locale à l'infini, est donnée sur $S$, sous laquelle $M$ est injective, c.a.d., $M f=0 \Longrightarrow f=0$. Le résultat d'injectivité est généralisé dans le cas où la transformée de Fourier de $f$ est quasi-analytique, de façon à ne pas supposer que f est à support compact. Une condition suffisante sur $S$ est donnée sous laquelle $M^{-1}$ peut être construit analytiquement. Deux exemples de formules d'inversion sont donnés : dans le cas où $S$ est plan, et dans le cas où $S$ est une sphère. Ces formules peuvent etre utilisés dans les applications. (c) 2002 Académie des sciences/Éditions scientifiques et médicales Elsevier SAS

Note présentée par Bernard MALGRANGE

S0764-4442(00)0-/FLA 


\section{Running authors}

Version française abrégée

Soit $S$ une surface de $\mathbb{R}^{n}$ qui divise l'espace en deux composantes connectées $D_{1}$ and $D_{2}$. Soit $f \in$ $C_{0}^{\infty}\left(\mathbb{R}^{n}\right)$ une fonction à valeurs réeles, supp $f \subset D_{1}$.

Considérons

$$
M f:=m(y, r):=\int_{\mathbb{R}^{n}} f(z) \delta(|y-z|-r) d z,
$$

où $\delta$ est la delta-fonction, $y \in S$ et $r>0$ sont quelconques. Une condition générale, locale à l'infini, est donnée sur $S$, sous laquelle $M$ est injective, c.a.d., $M f=0 \Longrightarrow f=0$. Le résultat d'injectivité est généralisé dans le cas où la transformée de Fourier de $f$ est quasi-analytique, de façon à ne pas supposer que $f$ est à support compact.

Une condition suffisante sur $S$ est donnée sous laquelle $M^{-1}$ peut être construit analytiquement. Deux exemples de formules d'inversion sont donnés : dans le cas où $S$ est plan, et dans le cas où $S$ est une sphère. Ces formules peuvent etre utilisés dans les applications.

Nos résultats sont formulés dans Théoremes 1.1 et 1.2.

Théorème 1.1. Si S est Lipschitzienne, $S_{R}$ est en forme d'étoile par rapport à un point $O \in D_{1}, f \in$ $C_{0}\left(\mathbb{R}^{n}\right)$, supp $f:=D \subset D_{1}$, et $M f=0$, i.e., $m(y, r)=0 \forall y \in S, \forall r>0$, alors $f=0$.

Théorème 1.2. (Ramm [8]) Sous l'hypothèse sur $S$ dans Théorème 1.1 le problème :

$$
\begin{gathered}
\left(\Delta+k^{2}\right) u=0 \quad \text { in } D_{2}, \quad k=\text { const }>0, \quad u=0 \quad \text { on } S, \\
\lim _{R \rightarrow \infty} \int_{|z|=R, z \in D_{2}}\left|\frac{\partial u}{\partial|z|}-i k u\right|^{2} d s=0,
\end{gathered}
$$

n'a q'une solution triviale $u=0$.

Théorème 1.1 reste valid pour des fonctions $f$ à décroissance suffisamment rapide, par exemple, pour $f$ avec transformée de Fourier $\tilde{f}$ une fonction entière. Un exemple d'une telle fonction $f$ est $|f(z)| \leq$ $c_{1} e^{-c_{2}|z|^{b}}$, où $b>1$ et $c_{j}>0$ sont des constants, $j=1,2$. Alors, il n'est pas nécessaire de supposé que $f$ est à support compact pour $M$ soit injective : $M$ est injective si $\tilde{f}$ est quasianalytique.

\section{Introduction}

In some physical problems and problems of mechanics it is of interest to know if the integrals of a function $f$ over a family of spheres of all radii $r>0$, with centers $y$ running through a given surface $S$, determine the $f$ uniquely. These integrals are called the spherical means of $f$. It is also of interest to have inversion formulas, which allow one to calculate $f$, given its spherical means.

In this paper the injectivity of the operator $M$ of the spherical means is studied for a wide class of surfaces, and inversion formulas are derived. These formulas are obtained by a method different from the one used in [1-4]. The injectivity result we obtain, is based on the results in [6], see also [7-9].

Let $S$ be a Lipschitz surface which divides the space $\mathbb{R}^{n}$ into two connected components, $D_{1}$ and $D_{2}$. By $S_{R}$ we denote the part of $S$ which is lying outside of the ball $B_{R}$, centered at a point $O \in D_{1}$, of radius $R$, where $R>0$ is an arbitrary large fixed number.

We assume that: $S_{R}$ is star-shaped with respect to $O$.

This assumption is local at infinity: it does not restrict $S$ inside $B_{R}$, apart of the Lipschitz smoothness of $S$.

Let $f$ be a real-valued compactly supported continuous function, $f \in C_{0}\left(\mathbb{R}^{n}\right)$, with supp $f:=D \subset D_{1}$. This assumption is relaxed below: it is sufficient to assume that the Fourier transform $\tilde{f}$ of $f$ is quasianalytic, so that if $\tilde{f}=0$ on an open set, then $\tilde{f}=0$ everywhere, and so $f=0$. 


\section{Running title}

Let $y \in S$ be an arbitrary point. Define the spherical means of $f$ by the formula:

$$
M f:=m(y, r)=\int_{\mathbb{R}^{n}} f(z) \delta(|y-z|-r) d z, \quad y \in S, r>0,
$$

where $\delta$ is the delta function, $r>0$ is arbitrary, and $y$ runs through the whole $S$. The injectivity problem we study is:

Problem I: Does $M f=0$ imply $f=0$ ?

This problem has been discussed in the literature for $S$ being a plane, a sphere, etc, [1-4], [6], [7]. Inversion formulas, which allow one to calculate $f$ given $m(y, r)$ for all $y \in S$ and all $r>0$, were obtained for the above cases. Some of these formulas ([3, pp. 754-756], [4, pp. 170-175]) are not suitable for practical calculations, and some are ([6], [7], [2]). The goal of this paper is to give a fairly general condition on $S$ sufficient for $M$ to be injective. In Section 2 we give some analytical inversion formulas for $M$.

The method we use is developed in [6]. In the proof of our main injectivity result, Theorem 1.1, an essential role is played by Theorem 1.2 of Ramm ([8], [9], [6, pp. 292, 296]), which we formulate below. This theorem is our main technical tool. Our main result is Theorem 1.1:

Theorem 1.1. If $S$ is a Lipschitz, $S_{R}$ is star-shaped with respect to a point $O \in D_{1}, f \in C_{0}\left(\mathbb{R}^{n}\right)$, supp $f:=D \subset D_{1}$, and $M f=0$, that is, $m(y, r)=0$ for all $y \in S$ and all $r>0$, then $f=0$.

See also Remark 1.3 below, in which the assumption of the compactness of the support of $f$ is discarded.

Theorem 1.2. (Ramm [8]) Under the assumptions on $S$ in Theorem 1.1, the problem:

$$
\begin{gathered}
\left(\Delta+k^{2}\right) u=0 \quad \text { in } D_{2}, \quad k=\text { const }>0, \quad u=0 \quad \text { on } S, \\
\lim _{R \rightarrow \infty} \int_{|z|=R, z \in D_{2}}\left|\frac{\partial u}{\partial|z|}-i k u\right|^{2} d s=0,
\end{gathered}
$$

has only the trivial solution $u=0$.

Let $g=g(x, z, k)$ be the unique solution to the problem:

$$
\left(\Delta+k^{2}\right) g=-\delta(x-z) \quad \text { in } \mathbb{R}^{n}, \quad k=\text { const }>0
$$

which satisfies the radiation condition (3). It is known that

$$
g(x, z, k):=\frac{i}{4} \frac{k^{\frac{n-2}{2}} H_{\frac{n-2}{2}}(k|x-z|)}{(2 \pi k|x-z|)^{\frac{n-2}{2}}} .
$$

We need the following formula, which can be obtained from (5) easily:

$$
g(x, z, k)=g(x, 0, k) e^{-i k\left(x^{0}, z\right)}+o\left(\frac{1}{|x|^{\frac{n-1}{2}}}\right),
$$

as $|x| \rightarrow \infty, x^{0}:=\frac{x}{|x|}$. A similar formula for the resolvent kernel of the Schrödinger operator was proved in [8], (see also [6, pp 45-46], where formula (6) was proved, and a similar formula for the resolvent kernel of the Laplacian in domains with boundaries was established, with the plane wave replaced by the scattering solution).

Theorem 1.1 includes many of the known to the author results on the injectivity of $M$ as very particular cases.

Remark 1.3: Our proof of Theorem 1.1 remains valid for sufficiently rapidly decaying functions $f$, for example, for such $f$ that their Fourier transform $\tilde{f}$ is an entire function. An example of such $f$ is $|f(z)| \leq c_{1} e^{-c_{2}|z|^{b}}$, where $b>1$ and $c_{j}>0$ are constants, $j=1,2$. Thus, it is not necessary to assume $f$ compactly supported for $M$ to be injective. It is sufficient for the injectivity of $M$ that $\tilde{f}$ is quasianalytic. 


\section{Running authors}

Often an analytical inversion formula can be constructed for $M$ if the problem:

$$
\left(\Delta+k^{2}\right) u=0 \quad \text { in } D_{2}, \quad k=\text { const }>0, \quad u=F(y, k) \quad \text { on } S,
$$

where $u$ satisfies (3), and $F(y, k)$ is a known rapidly decaying function, can be analytically solved. In [5, p.655] the coordinate systems in which the operator $\Delta+k^{2}$ admits separation of variables are listed. If $S$ is a coordinate surface (curve if $n=2$ ) of one of these coordinate systems, then problem (7)-(3) can be solved analytically, and an analytical inversion formula for $M$ can be obtained. Planes and spheres are particular cases of such coordinate surfaces, and examples of inversion formulas for $M$ are given for these cases in Section 2. The method used in Section 2, is the basis for the argument in Section 3, where Theorem 1.1 is proved.

In [7, p.317-320] the following problem of integral geometry is solved analytically: Given $\mathcal{H}(x)=$ $\int_{S^{2}} h(x+|x| \alpha) d \alpha$ for all $x \in \mathbb{R}^{3}$, find $h(x)$.

\section{Auxiliary results}

2.1.

In this section we prove injectivity of $M$ and derive analytical inversion formulas in the case when $S$ is a plane or a sphere. For simplicity of writing we take $n=3$. Our proofs are valid with trivial modifications for any $n \geq 2$. We follow the method developed in [6, p.244].

Theorem 2.1. Let $S$ be a plane $\left\{x: x_{3}=0\right\}$, and supp $f \subset \mathbb{R}_{-}^{3}:=\left\{z: z_{3}<0\right\}$ be compact. Then $M$ is injective and $M^{-1}$ can be computed by inverting the Fourier transform of $f$ given by formula (14) below.

Proof. Multiply (1) by $\frac{e^{i k r}}{4 \pi r}$ and integrate over $[0, \infty)$ with respect to $r$ to get:

$$
\int_{\mathbb{R}^{3}} \frac{e^{i k|y-z|}}{4 \pi|y-z|} f(z) d z=\mu(y, k),
$$

where

$$
\mu(y, k):=\int_{0}^{\infty} \frac{m(y, r)}{4 \pi r} e^{i k r} d r,
$$

is the known function for all $y \in S$ and all $k>0$. Fourier transform (8) with respect to two-dimensional variable $y$ over the plane $\mathbb{R}^{2}$ to get:

$$
\int_{\mathbb{R}^{2}} d y e^{i \xi^{1} \cdot y} \int_{\mathbb{R}^{3}} d z \frac{e^{i k|y-z|}}{4 \pi|y-z|} f(z)=F(\xi)
$$

where

$$
\xi^{1}:=\left(\xi_{1}, \xi_{2}\right), \quad \xi:=\left(\xi^{1},-\xi_{3}\right), \quad \xi_{3}:=\left(k^{2}-\left|\xi^{1}\right|^{2}\right)^{\frac{1}{2}}
$$

and

$$
F\left(\xi^{1}, k\right):=\int_{\mathbb{R}^{2}} d y e^{i \xi^{1} \cdot y} \mu(y, k) d y
$$

is a known function $\forall \xi^{1} \in \mathbb{R}^{2}$ and $\forall k>0$. Using the formula [6, p.244]:

$$
\int_{\mathbb{R}^{2}} \frac{e^{i k|y-z|}}{4 \pi|y-z|} e^{i \xi^{1} \cdot y} d y=\frac{i e^{i\left(\xi^{1} \cdot z^{1}+\left|z_{3}\right| \xi_{3}\right)}}{2 \xi_{3}}, \quad k^{2} \geq\left|\xi^{1}\right|^{2}
$$

and taking into account that $\left|z_{3}\right|=-z_{3}$, because supp $f \subset \mathbb{R}_{-}^{3}:=\left\{z: z_{3}<0\right\}$, one calculates the left-hand side of (10) and gets:

$$
\tilde{f}(\xi):=\int_{\mathbb{R}^{3}} e^{i \xi \cdot z} f(z) d z=-2 i \xi_{3} F\left(\xi^{1}, k\right), \quad k^{2} \geq\left|\xi^{1}\right|^{2}
$$




\section{Running title}

Since the right-hand side of (14) is known for all $\xi^{1} \in \mathbb{R}^{2}$ and all $k>0$, formula (14) defines the Fourier transform of $f$ for all $\xi^{1} \in \mathbb{R}^{2}$ and all $\xi_{3} \geq 0$. Since $f$ is real-valued, $\tilde{f}(-\xi)=\bar{f}(\xi)$, where the overbar stands for complex conjugate, so $\tilde{f}$ is defined everywhere in $\mathbb{R}^{3}$. Thus, $f$ can be obtained uniquely by the Fourier inversion of $\tilde{f}$.

\section{2.}

Assume now that $S$ is a sphere of radius $a$, and $\operatorname{supp} f \subset B_{a}:=\{z:|z| \leq a\}$. The problem is: given $m(y, r)$ for all $y \in S$ and all $r>0$, find $f$.

The method for solving this problem is similar to the one in Section 2.1. Therefore we outline it briefly.

Theorem 2.2. Let $S$ be a sphere of radius a, centered at the origin, and supp $f \subset B_{a}$. Then $M$ is injective and $M^{-1}$ can be computed by formulas (2.9) and (2.10) below.

Proof. One gets formula (8), as above. Then one uses eigenfunction expansion of the Green function:

$$
\frac{e^{i k|y-z|}}{4 \pi|y-z|}=i k \sum_{\ell=0}^{\infty} \bar{Y}_{\ell}\left(z^{0}\right) Y_{\ell}\left(y^{0}\right) j_{\ell}(k|z|) h_{\ell}(k a), \quad z^{0}:=\frac{z}{|z|}, \quad|z|<|y|=a,
$$

where $Y_{\ell}=Y_{\ell m},-\ell \leq m \leq \ell$, are orthonormal spherical harmonics, $\sum_{\ell=0}^{\infty}=\sum_{\ell=0}^{\infty} \sum_{m=-\ell}^{\ell}, j_{\ell}(r)=$ $\sqrt{\frac{\pi}{2 r}} J_{\ell+\frac{1}{2}}(r)$ is the spherical Bessel function, and $h_{\ell}(r)=\sqrt{\frac{\pi}{2 r}} H_{\ell+\frac{1}{2}}^{(1)}(r)$ is the spherical Hankel function (see e.g., [6, p.140]). Let

$$
f(z)=\sum_{\ell=0}^{\infty} Y_{\ell}\left(z^{0}\right) f_{\ell}(|z|)
$$

where $f_{\ell}(|z|)$ are the Fourier coefficients of $f(z)$ with respect to the spherical harmonics.

Substitute (15) and (16) into (8), use the orthonormality of the spherical harmonics, and get:

$$
\int_{0}^{\infty} f_{\ell}(s) j_{\ell}(k s) s^{2} d s=\left[i k h_{\ell}(k a)\right]^{-1} \mu_{\ell}(k), \quad s:=|z|
$$

where

$$
\mu_{\ell}(k):=\int_{S^{2}} \mu\left(a y^{0}, k\right) \bar{Y}_{\ell}\left(y^{0}\right) d y^{0},
$$

and we do not show the dependence of $\mu_{\ell}$ on $a$.

Formula (17) is the Bessel transform of $f_{\ell}(s)$, and it can be inverted analytically for $f_{\ell}(s)$ for every $\ell=0,1,2, \ldots$. Thus, all the Fourier coefficients $f_{\ell}(|z|)$ of $f$ are recovered, and $f$ is recovered by formula (16).

Recall that the standard inversion formulas for the Bessel transform with an arbitrary positive index $\ell$ are:

$$
H(k):=\int_{0}^{\infty} h(s) J_{\ell}(k s) s d s, \quad h(s)=\int_{0}^{\infty} H(k) J_{\ell}(k s) k d k .
$$

Theorem 2.2 is proved.

\section{Proof of Theorem 1.1 .}

Multiply (1) by $g(k r, k):=\frac{i}{4} \frac{k^{\frac{n-2}{2}} H_{\frac{n-2}{2}}(k r)}{(2 \pi k r)^{\frac{n-2}{2}}}$, integrate with respect to $r$ over $[0, \infty)$, and get an analog of (8):

$$
w(y, k):=\int_{D} g(y, z, k) f(z) d z=\int_{0}^{\infty} m(y, r) g(k r, k) d r:=F(y, k),
$$




\section{Running authors}

where

$$
w(x, k):=\int_{D} g(x, z, k) f(z) d z, \quad x \in \mathbb{R}^{n},
$$

solves equation (7) in $D_{2}$, satisfies the radiation condition (8) in $D_{2}$, and takes the value $F(y, k)$ on $S$. To prove the injectivity of $M$, it is sufficient to prove that problem (7)-(8) with $F(y, k)=0$ has only the trivial solution, and this is exactly the claim of Theorem 1.2.

Indeed, if problem (7)-(8) with $F(y, k)=0$ has only the trivial solution, then function (19) solves equation (7) everywhere outside the support $D$ of $f$, vanishes in $D_{2}$, and by the unique continuation property for solutions of elliptic equations, it vanishes outside $D$. Thus, using formula (1.6), one sees that the Fourier transform of $f$ vanishes, because $x^{0}$ is arbitrary in $S^{n-1}$, the unit sphere in $\mathbb{R}^{n}$, and $k>0$ is arbitrary. Thus $f=0$, and the injectivity of $M$ is proved. Theorem 1.1 is proved.

After this paper was written, Professors Quinto and Agranovsky informed me about their recent works on the injectivity of $M$. Their interesting results do not contain the results of this paper, and our method of proof is completely different from theirs [1].

\section{References}

[1] Agranovsky, M., Quinto, E., Injectivity of the spherical mean operator and related problems, Pitman Res. Notes Math. Ser., 347, Longman, Harlow, 1996, pp. 12-36.

[2] Anderson, L., On the determination of the function from spherical averages, SIAM J. of Math.Anal., 19, (1988), 214-232.

[3] Courant, R., Hilbert, D., Methods of mathematical physics, Interscience, New York, 1962.

[4] Lavrent'ev, M., Romanov, V., Shishatskii, S., Ill-posed problems of mathematical physics and analysis, Amer. Math. Soc., Providence, RI, 1986.

[5] Morse, P., Feshbach, H., Methods of theoretical physics, McGraw Hill, New York, 1953.

[6] Ramm, A.G., Scattering by obstacles, Reidel, Dordrecht, 1986, pp.1-442.

[7] Ramm, A.G., Multidimensional inverse scattering problems, Longman Scientific \& Wiley, New York. 1992, pp.1385; Expanded Russian edition, Mir, Moscow, 1994,pp.1-496.

[8] Ramm, A.G., Spectral properties of the Schrödinger operator in some domains with infinite boundaries. Soviet math. Doklady, 152, (1963) 282-285.

[9] Ramm, A.G., Spectral properties of the Schrödinger operator in some infinite domains, Matem. Sbornik, 66, (1965), 321-343. 